Revue d'histoire de l'enfance « irrégulière »

Le Temps de l'histoire

Hors-série | 2007

Pages d'histoire, la protection judiciaire des mineurs, $\mathrm{XIX} \mathrm{X}^{\mathrm{e}} \mathrm{XX} \mathrm{X}^{\mathrm{e}}$ siècles

\title{
Saint-Maurice, colonie pénitentiaire agricole
}

Le temps de la réforme : 1934-1936-1950

Jacques Bourquin

\section{OpenEdition}

\section{Journals}

Édition électronique

URL : http://journals.openedition.org/rhei/3023

DOI : $10.4000 /$ rhei.3023

ISBN : 978-2-7535-1647-2

ISSN : 1777-540X

Éditeur

Presses universitaires de Rennes

Édition imprimée

Date de publication : 1 juin 2007

Pagination : 267-281

ISSN : $1287-2431$

\section{Référence électronique}

Jacques Bourquin, «Saint-Maurice, colonie pénitentiaire agricole », Revue d'histoire de l'enfance "

irrégulière » [En ligne], Hors-série | 2007, mis en ligne le 01 février 2010, consulté le 19 avril 2019. URL : http://journals.openedition.org/rhei/3023 ; DOI : 10.4000/rhei.3023 


\section{Saint-Maurice,}

\section{colonie pénitentiaire agricole}

Le temps de la réforme :

\section{4-1936-1950 ${ }^{(*)}$}

Le journal Paris Soir du dimanche 10 janvier 1937 titre sur trois colonnes à

$\left.{ }^{*}\right)$ Article publié

la Une : «Vingt jeunes instituteurs volontaires vont prendre la place des surveillants dans ce qui fut les bagnes d'enfants. La prison de Lamotte-Beuvron sera le ler février une vraie maison d'éducation. "

\section{Les campagnes de presse contre les bagnes d'enfants, 1934-1937}

Ce titre bien optimiste et accrocheur pour le public introduit l'article d'un journaliste connu, Alexis Danan qui, depuis 1934, mène dans la presse et dans les cabinets ministériels une campagne rigoureuse et justifiée contre les établissements pour mineurs gérés par l'Administration pénitentiaire. Ce terme de bagnes d'enfants est depuis le début du siècle de plus en plus utilisé pour évoquer ces institutions dont fait partie Saint-Maurice.

Pourtant en 1927, les colonies pénitentiaires pour mineurs avaient changé de nom, on les appela alors : maisons d'éducation surveillée. Les surveillants avaient échangé leur titre contre celui de moniteur, les colons étaient devenus des pupilles, mais tous ces changements avaient été de pure forme, les murs, les règlements, les méthodes, les quartiers disciplinaires étaient restés ce qu'ils étaient au XIXème siècle.

En devenant pupilles, les colons étaient restés des "inamendables ", une seule chose avait changé pour eux, ils ne portaient plus des sabots mais des galoches. Quant aux surveillants devenus moniteurs, ils continuaient à surveiller, on ne leur avait donné aucune formation nouvelle, là aussi le changement n'avait été que vestimentaire : ils avaient abandonné le képi pour le remplacer par une casquette. Réforme dérisoire pour ces " pupilles " dont Louis Roubaud écrivait en 1925 dans Les enfants de Caïn, une enquête sur les colonies pénitentiaires : dans Le Journal de la Sologne, $\mathrm{n}^{\circ} 83$, janvier 1994

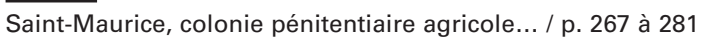


(1) Louis Roubaud, Les enfants de Caïn, Paris, Grasset, 1925.
" Je viens de trouver, réunis dans des garderies spéciales, des enfants qui ne seront jamais des hommes. Après les avoir retranchés du monde, on les prépare à n'y jamais revenir, ce sera leur carrière d'être morts. » ${ }^{(1)}$

Dans les maisons d'éducation surveillée des années 1930, l’ordre et la discipline, hors de tout projet d'éducation, continuaient à régner dans une atmosphère d'expiation.

En août 1934, une révolte éclate à la maison d'éducation surveillée de Belle-Île-en-Mer, un établissement pour mineurs ouvert sur une île en 1880.

D'autres incidents éclateront dans d'autres colonies. L'opinion publique s'émeut, d'autant plus qu'à cette époque la délinquance juvénile ne fait pas peur, le chiffre n'en a jamais été aussi bas depuis le début du siècle, ce qui s'explique en grande partie par le fait qu'il y a eu très peu de naissances entre 1914 et 1918 donc peu d'adolescents dans les années 1930. Très vite, l'enfant des bagnes que l’on découvre inspire surtout pitié, il émeut, on le considère plus comme une victime que comme un coupable.

Le pouvoir politique se saisit de l'affaire, un groupe parlementaire est constitué autour de ce problème de l'enfance délinquante. On reparle de réformes, on change le directeur de l'Administration pénitentiaire et le 13 août 1936, le garde des Sceaux du gouvernement du Front populaire, Marc Rucart, fait voter par le parlement une loi relative à la réforme de la maison d'éducation surveillée de Saint-Maurice à Lamotte-Beuvron. Si l'on choisit cette institution, c'est qu'il semble qu'elle soit la plus réformable et peut-être une des moins répressives. Les campagnes de presse prendront fin en 1937 suite à un incident très grave à Eysses où un mineur meurt après 90 jours de cachot.

Deux établissements seront fermés définitivement : la colonie privée de Mettray en 1937 et l'ancienne colonie publique d'Eysses en 1940.

\section{La première réforme de Saint-Maurice, 1936-1937}

En mars 1936, l'Administration pénitentiaire avait demandé à un inspecteur général de l'Enseignement technique, J. Roumajon, de bien vouloir visiter la maison d'éducation surveillée de Saint-Maurice. Il est à remarquer que cette inspection est confiée à une personnalité qui ne relève pas du ministère de la Justice, ce qui est une innovation. Son rapport nous apprend qu'il y a 226 pupilles à Saint-Maurice. Parmi eux, 4/5 sont originaires de la ville, 2/3 tra- 
vaillent à la ferme et seulement 43 sont affectés dans des ateliers artisanaux (scierie, ferblanterie, menuiserie, forge, cordonnerie).

Dans ses conclusions, l'inspecteur général Roumajon est très sévère :

« La maison de Saint-Maurice est écrasée par le souci de gérer l'exploitation d'un domaine trop vaste.

"Ni les personnels ni les élèves ne prennent intérêt à ce qu'ils font. Aucun des surveillants ou ouvriers n'est capable de diriger un apprentissage. Les préoccupations principales se limitent à :

- « éviter les évasions,

- « prévenir les faits sexuels,

- « occuper les pupilles et les moniteurs à tous les moments de la journée. " (2)

On a l'impression à la lecture du rapport que pupilles et moniteurs sont inscrits dans les mêmes dépendances. La conclusion générale est que SaintMaurice « donne l'impression d'un long stage pénalisateur exigé par une fatalité inéluctable, sans autre utilité que sa durée ». ${ }^{(3)}$

J. Roumajon propose de :

- «Renoncer à l'exploitation de la ferme et du domaine.

- «Faire de Saint-Maurice une institution consacrée à l'apprentissage de métiers bien choisis.

- «Recevoir des adolescents dont la volonté de redressement est manifeste d'où la nécessité d'écarter les déficients physiques et mentaux, de sélectionner les personnels, de faire disparaître des traditions regrettables (il s'agit vraisemblablement d'onanisme et d'homosexualité que l'on retrouve dans beaucoup d'établissements de ce type).

- "Choisir des métiers à caractère suffisamment généraux.

- "Prévoir une durée d'apprentissage de 2 à 3 ans. " ${ }^{(4)}$

La direction de l'Enseignement technique est prête à collaborer à ce projet. Les choses vont aller vite, dès la loi du 13 août 1936. Le garde des Sceaux associe à la réforme de Saint-Maurice la direction de l'Enseignement technique qui, à l'époque, dépend du ministère du Travail, et la direction de l'Enseignement primaire à l'Éducation nationale.

À l'une et à l'autre, la direction de l'Administration pénitentiaire va demander des personnels. Un cadre de l'Enseignement technique est nommé à la tête de Saint-Maurice pendant le dernier trimestre 1936, il remplace le direc-

\section{(2) J. Roumajon,}

Rapport d'inspection sur la maison d'éducation surveillée de SaintMaurice, mars 1936, document consulté à la bibliothèque $2 \mathrm{RJ}$, CNFE-PJJ, Vaucresson.

(3) Ibid.

(4) Ibid. 
teur du cadre pénitentiaire. Plusieurs instructeurs de l'Enseignement technique l'accompagnent. L'Éducation nationale sollicitée ne cède qu'avec parcimonie, à la fin de l'année 1936, une vingtaine d'instituteurs dont la majorité sont surnuméraires et n'ont de ce fait que très peu d'expérience pédagogique. Ils sont toutefois volontaires, on va les recruter en tant que moniteuréducateur. Le scoutisme est aussi sollicité. L’Administration pénitentiaire va demander à Jacques Guérin-Desjardins, commissaire général des Éclaireurs de France, de bien vouloir former ces premiers moniteurs-éducateurs au cours d'un stage d'un mois qui aura lieu à... la prison de Fresnes.

Les nouvelles recrues reçoivent quelques rudiments de droit, de psychologie, de pédagogie à base de scoutisme. Ils passent quelques jours en Belgique à Möli, une institution où, depuis le début du siècle, on s'occupe de manière pédagogique de mineurs délinquants.

Les moniteurs-éducateurs arrivent à Saint-Maurice en janvier 1937, les personnels pénitentiaires en place n’ont nullement été associés à la réforme malgré la nouvelle direction. Ce sera très vite un échec cuisant pour les malheureux moniteurs-éducateurs. On ne passe pas impunément d'une "pédagogie pénitentiaire » à la pédagogie scoute sûrement peu adaptée. On ne transforme pas en quelques semaines un groupe d'épreuve en patrouille de boy-scouts !

Les moniteurs-éducateurs qui ont remplacé tous les instituteurs pénitentiaires sauf trois sont vite débordés. De nombreux élèves s'évadent ou se rebellent, il y aura une tentative de révolte au réfectoire en octobre 1937.

Malgré cela, durant cette période, des réformes se réalisent à Saint-Maurice. On détruit le quartier cellulaire et on construit à sa place deux grands ateliers fer et bois. On installe le chauffage central dans les classes et dans les ateliers. On abat le mur d'enceinte construit par les colons depuis la fin du siècle dernier et jamais terminé. On modernise les cages à poules, mais elles ne sont pas abandonnées.

Henri Gaillac donne plusieurs raisons à cet échec :

"La campagne contre les bagnes d'enfants se poursuit, elle a des répercussions sur les établissements.

"Les méthodes du scoutisme, telles qu'elles sont appliquées ne sont pas adaptées à des enfants difficiles qui sont là depuis des années. Les instituteurs n'ont aucune expérience. Les personnels pénitentiaires sont plus ou 
moins goguenards et voient l'expérience d'un mauvais œil.

«Quant au nouveau directeur qui vient de l'Enseignement technique, il est en conflit avec les personnels pénitentiaires et les moniteurs-éducateurs. " ${ }^{(5)}$ En février 1937, une seconde inspection est demandée par la direction de l'Administration pénitentiaire à J. Roumajon. Le constat est aussi sévère que celui de l'année précédente :

«Pas de cohésion, pas d'esprit d'ensemble. On a changé les hommes, mais l'esprit est resté le même. L'orientation professionnelle des pupilles n'a pas été faite en fonction des métiers enseignés. " ${ }^{(6)}$

Suite à ce premier échec, un inspecteur général de l'Administration, Jean Bancal, écrit : "Il serait prudent pour l'Administration pénitentiaire, avant de nommer des cadres pédagogiques extérieurs, de ménager une période transitoire pendant laquelle les fonctions de directeur et sous-directeur soient réservées à des personnels issus de ses propres cadres. " ${ }^{(7)}$

\section{La seconde réforme de Saint-Maurice, juin 1938}

Les conseils de l'inspecteur général Bancal vont être suivis. L'Administration pénitentiaire nomme le 20 mai 1938 deux de ses fonctionnaires à la tête de Saint-Maurice. Comme directeur : Vincent Hourcq, un cadre administratif. Comme sous-directeur : René Courtois qui a été entre 1929 et 1931 instituteur à l'école de réforme de Saint-Hilaire, un établissement pénitentiaire pour mineurs.

Les deux hommes arrivent le 6 juin à Saint-Maurice, l'un et l'autre ont travaillé ensemble à la prison de Fontevraud, ils se connaissent bien, ont des idées communes et sont tous deux très favorables au projet Roumajon.

Vincent Hourcq écrit dans un rapport de 1938 :

«On ne peut dire que les principes de l'éducation des pervertis, des délinquants soient différents de ceux de l'éducation des enfants normaux. Ils sont souvent les mêmes et ne varient que dans leur application. Ils exigent de l'éducateur une souplesse avertie, alliée à une autorité plus affirmée ainsi que l'observation de certaines règles et de mesures de précaution. " ${ }^{(8)}$ Le propos est intéressant, en ce qu'il réintroduit le mineur délinquant dans le champ d'une certaine normalité. Le rapport Hourcq va à l'encontre des théories médicales de l'époque très influencées par le courant de la neuro-

(5) Henri Gaillac, Les maisons de correction, Paris, Éd. Cujas, rééd. 1991.

(6) Jean Roumajon, Rapport d'inspection sur la maison d'éducation surveillée de SaintMaurice, février 1937 , document consulté à la bibliothèque 2RJ, CNFE-PJJ, Vaucresson.

(7) Jean Bancal, Rapport sur le fonctionnement des personnels éducatifs et pénitentiaires, Imprimerie administrative de Melun, 1938, p. 119.

(8) Cité par Philippe Rey-Herme, in Quelques aspects du progrès pédagogique dans la rééducation de la jeunesse délinquante, Paris, Vrin, 1945. 
psychiatrie infantile qui considère plus ou moins les enfants délinquants comme victimes de tares héréditaires.

En fait, pour Vincent Hourcq, la maison d'éducation surveillée doit plus se rapprocher de l'école que de l'hôpital ou de la prison.

C'est ce projet dont Roumajon fut le théoricien et Hourcq et Courtois les maîtres d'œuvre qui déterminera en 1938-1939 ce que seront les internats de rééducation pour délinquants de l'après-guerre.

Vincent Hourcq et René Courtois vont associer les personnels pénitentiaires de l'institution à la réforme, ils ne retiendront que les volontaires. Les enseignants techniques recrutés entre 1936 et 1937 sont maintenus sous la direction de M. Rouault, un ingénieur des Arts et Métiers. Quelques moniteurs-éducateurs seront embauchés. Sur 226 jeunes placés à Saint-Maurice en 1936, il en reste 170 en juin 1938, on n’a pas conservé ceux qui étaient depuis trop longtemps dans l'institution et pour lesquels la réforme intervenait trop tard.

Dès juillet 1938, on présente des élèves au CEP ; contrairement aux années précédentes, il y a un nombre important de réussites. Le CAP organisé en 1939 avec le concours de l'ENP de Vierzon verra 15 réussites de jeunes de Saint-Maurice sur 15 présentés. Lorsqu'on porte sur ces jeunes un autre regard, les résultats changent.

Certes, de nombreuses habitudes pénitentiaires subsistent. Le quartier cellulaire détruit en 1936 a été remplacé par un quartier d'isolement beaucoup plus modeste : une demi-douzaine de cellules.

Pour les " élèves " les plus méritants, on ne parle plus de pupilles, il est prévu en 1939 un groupe dit "super mérite » qui fonctionne en semi-autonomie sans moniteur-éducateur. On commence à parler de pédagogie fondée sur le self government d'inspiration anglaise, mais tout cela est encore embryonnaire et fragile.

Le 25 octobre 1938, le garde des Sceaux, Paul Reynaud, signe une circulaire relative à l'emploi du temps des maisons d'éducation surveillée qui s'applique surtout à Saint-Maurice et Saint-Hilaire, les deux seules institutions en cours de réforme. Cette circulaire prévoit 22 heures de culture physique par semaine, n'oublions pas que le sport pour les masses est une des conquêtes récentes du Front populaire ; à cela s'ajoutent 30 heures d'enseignement scolaire et professionnel et des veillées-causeries chaque soir. Les journées sont 
longues! Il s’agit aussi d'occuper les jeunes ! Les élèves placés à l'isolement bénéficieront eux aussi de 2 heures d'éducation physique par jour avec un moniteur.

Vincent Hourcq évoquant à cette époque la formation morale des élèves de Saint-Maurice confie à Philippe Rey-Herme :

«La morale est action ou n'est rien. L'homme se forme moralement par des actes, chaque acte facilitant l'acte qui suit jusqu'à ce que bien agir devienne non seulement une habitude mais une joie. " ${ }^{(9)}$

(9) Ibid.

Toute une pédagogie qui commence à se centrer sur la vie de groupe doit leur permettre "d'apprendre au sein de cette petite société de bonnes habitudes de vie... " ${ }^{(10)}$ Ce début de réforme est stoppé par la guerre.

\section{Le temps de la guerre}

En juin 1940, Saint-Maurice est replié sur l'établissement correctif pour mineurs d'Aniane près de Montpellier. Cet établissement n'a fait l'objet d'aucune réforme, il a de surcroît la caractéristique de recevoir les mineurs les plus durs. C'est un spectaculaire retour en arrière qui durera jusqu'au retour à LamotteBeuvron en août 1941. René Courtois, prisonnier de guerre, ne revient à SaintMaurice qu'en avril 1941. Avec Vincent Hourcq, tous deux vont reprendre l'œuvre entamée.

Pendant cette période de guerre, l'effectif de l'institution est d'environ 240 élèves auquel il faut ajouter, pour la période qui va de 1942 à la Libération, 88 mineurs qui sont placés dans des familles rurales environnantes. En relation avec le médecin de l'institution qui connaît bien les familles de la région, Hourcq et Courtois arrivent à trouver sans difficultés des familles d'accueil, en permettant au projet de Saint-Maurice de s'ouvrir sur l'extérieur. Qui sont ces mineurs placés à Saint-Maurice pendant la guerre, dont certains ont eu par la suite de belles réussites sociales ? Ils sont à l'image même de la complexité de l'époque, de la délinquance des mineurs de la guerre, où le marché noir est une tentation évidente mais peut aussi être une manière de lutter contre l'occupant. Voler des marchandises aux Allemands n'est pas sans panache et parfois héroïsme.

Voici l'attendu d'un jugement de 1943 confiant un jeune à Saint-Maurice. Ce jeune avait saccagé un bureau d'embauche pour le STO. Le juge avait écrit comme attendu :

«Un bureau d'embauche pour l'Allemagne n'ayant pas un caractère social 
(11) Entretien de Jacques Bourquin avec René Courtois, septembre 1986 .

(12) Paul Lutz, Rapport d'inspection sur la maison d'éducation surveillée de SaintMaurice, novembre 1944, document consulté à la bibliothèque $2 \mathrm{RJ}$, CNFE-PJJ, Vaucresson.

(13) Rapport de René Courtois remis au sous-directeur de l'Education surveillée, 1944 , document consulté à la bibliothèque $2 \mathrm{RJ}$, CNFE-PJJ, Vaucresson.

(14) Ibid. et ne correspondant pas aux besoins du pays, je confie le jeune X... pour une mesure éducative à l'établissement d'éducation surveillée de SaintMaurice. " ${ }^{(11)}$

Il y aura aussi des enfants juifs confiés à Saint-Maurice par les magistrats et ceux-ci, dans le souci de les protéger des occupants, mentionnent comme motif de placement "fausse identité ».

Dans une inspection du 23 novembre 1944, le contrôleur général de l'Administration évoquant les 88 mineurs placés dans des familles depuis 1942 constatera qu'il y a eu 23 évasions au moment de la Libération. Il conclura, suite à sa rencontre avec René Courtois : "Le chiffre d'évasion est particulièrement élevé en raison du départ pour le maquis de beaucoup de jeunes placés. » ${ }^{(12)}$

On peut supposer que la direction de Saint-Maurice sut fermer les yeux.

Vincent Hourcq nommé à un autre poste, René Courtois prend la direction de Saint-Maurice en avril 1944. Au lendemain de la Libération, il rend un rapport ${ }^{(13)}$ au sous-directeur de l'Éducation surveillée et y évoque les événements contemporains de la libération à Saint-Maurice :

5 juillet 1944 : bombardement de la voie ferrée, un maître surveillant est tué à son domicile, quelques dégâts sont occasionnés à l'établissement.

8 juillet 1944 : bombardement de Lamotte-Beuvron.

12 juillet 1944 : bombardement de la gare. 19 août 1944 : arrivée des FTP.

20 août 1944 : arrivée des FFI, parmi eux deux élèves évadés dont l'un sera tué quelques jours plus tard dans les combats de la libération de Paris.

23 août 1944 : passage des troupes allemandes, les FFI décrochent. Les combats ont lieu en bordure de l'établissement. Les Allemands occupent quelques heures Saint-Maurice. Ils partent lorsqu'on leur dit qu'il s'agit d'une prison.

30 août 1944 : deux compagnies allemandes cantonnent dans l'établissement. Ils partent le 31 .

Dans ce document, René Courtois conclut en évoquant pendant toute cette période le courage des élèves :

"L'attitude des élèves pendant les bombardements, leur ardent désir de combattre m'encouragent. C'est je crois, chez une grande partie de nos jeunes délinquants que l'on trouve le plus d'idéal, lorsqu'on sait gratter délicatement la couche de crasse qui les recouvre. " ${ }^{(14)}$

La rééducation est en bonne voie ! 


\section{Laprès-guerre, "l'école de Saint-Maurice "}

Dans son rapport du 23 novembre 1944, le contrôleur général des services de l'Éducation surveillée rend hommage à l'œuvre menée par Vincent Hourcq et René Courtois pendant la guerre :

«La maison d'éducation surveillée de Saint-Maurice se place nettement en tête des établissements de rééducation. " ${ }^{(15)}$

À Paris, au lendemain de la Libération, il se passe des choses importantes pour l'Éducation surveillée. Le 2 février 1945, le gouvernement provisoire de la République promulgue une ordonnance concernant l'enfance délinquante. Cette ordonnance qui généralise les tribunaux pour enfants et crée le juge des enfants, affirme la notion d'éducabilité du mineur délinquant en considérant que la sanction pénale ne doit être qu'exceptionnelle. C'est un considérable changement par rapport aux législations précédentes. Cette ordonnance va tout à fait dans le sens des réformes dont Saint-Maurice est le modèle depuis 1938.

Quelques jours après cette ordonnance, Mme Delaunay, femme du préfet de Loir-et-Cher à la Libération, qui est une des douze femmes à siéger, en tant que résistante, à l'Assemblée consultative provisoire à Paris, ${ }^{(16)}$ fait devant ses collègues un discours en faveur de la réforme de l'Éducation surveillée. Mme Delaunay qui a visité Saint-Maurice à plusieurs reprises en fait l'illustration majeure dans son intervention :

"Il faut reconnaître que toutes nos maisons de redressement, ou si l'on veut dire, nos établissements d'éducation surveillée, n’ont pas atteint le même degré de perfectionnement, de modernisme que l'établissement de Saint-Maurice à Lamotte-Beuvron. " ${ }^{(17)}$

Au cours de son discours, fort applaudi, Mme Delaunay demande que l'Éducation surveillée soit détachée de l'Administration pénitentiaire et qu'elle devienne une direction autonome du ministère de la Justice spécialisée dans la rééducation des mineurs délinquants.

C'est le décret que prendra le gouvernement provisoire le 1er septembre 1945 en créant la direction de l'Éducation surveillée. Quelques mois auparavant a été créé un corps d'éducateurs qui remplace les moniteurs-éducateurs.

Le terme d'IPES (institution publique d'éducation surveillée) remplace celui de MES (maison d'éducation surveillée), mais très vite à Saint-Maurice on parlera de l'internat professionnel d'éducation surveillée, puis encore plus
(15) Paul Lutz, Rapport d'inspection, op. cit.

(16) Cette assemblée précède l'Assemblée constituante de 1946.

(17) Journal officiel du 21 février 1945. 
(18) Plan de réforme de l'Éducation surveillée, 1946, document consulté à la bibliothèque $2 \mathrm{RJ}$, CNFE-PJJ, Vaucresson.

(19) Conférence de René Courtois aux éducateurs sur « l'influence professionnelle en IPES ", Marly-le-Roi, novembre 1947, document consulté à la bibliothèque $2 \mathrm{RJ}$, CNFE-PJJ, Vaucresson.

(20) Conférence de René Courtois sur les "bases de la pédagogie spéciale des adolescents délinquants ", SaintMaurice, décembre 1955, document consulté à la bibliothèque $2 \mathrm{RJ}$, CNFEPJJ, Vaucresson.

(21) René Courtois, "Saint-Maurice, école d'apprentissage moderne ", Rééducation, $\mathrm{n}^{\circ} 1$, mars-avril 1946, p. 68.

(22) Ibid. simplement de l'école de Saint-Maurice. Il est clair que la référence est bien l'école et non la prison.

L'idée-force développée par la nouvelle direction de l'Éducation surveillée, c'est que la formation professionnelle est la base de toute rééducation :

"L'apprentissage, dit-on, non seulement concourt puissamment à donner aux jeunes le goût du travail, mais il accroît la volonté, la persévérance. C'est le meilleur moyen de reclassement social. Il fait du jeune délinquant un ouvrier indépendant matériellement, adapté à la vie sociale, capable de vivre normalement. " ${ }^{(18)}$

Dès 1945, les personnels pénitentiaires disparaissent progressivement. Autour de René Courtois et de M. Rouault, c'est une équipe d'instructeurs techniques, d'éducateurs, de personnels administratifs qui poursuivent en la développant la réforme entamée en 1937 et 1938.

\section{La formation professionnelle}

Elle est au cour du projet de Saint-Maurice comme des quelques IPES qui se mettent en place. Dans cette France dévastée qu'il s'agit de reconstruire, le projet pédagogique repose sur des valeurs qui sont fortes au lendemain de la guerre : le travail, l'amour du métier, et pour ces jeunes dont la grande majorité est issue de la classe ouvrière, l'appartenance à la classe ouvrière est à l'époque une valeur forte sur laquelle peut s'appuyer un projet de rééducation. René Courtois, qui a laissé de nombreux écrits qui sous-tendent le projet de Saint-Maurice, évoque :

"L'influence de l'ouvrier qui connaît parfaitement son métier est indiscutable sur nos adolescents. " ${ }^{(19)}$

Parlant plus particulièrement du mineur délinquant :

"Cet amour du métier l'aide à atténuer ses sentiments d'infériorité, ses réactions d'opposition et d'hostilité à l'adulte, à l'environnement... " (20)

Si le projet a une fonction de normalisation : "l'atelier, école d'énergie, de courage, de responsabilité, de conscience professionnelle permet d'intégrer l'image du bon ouvrier ", ${ }^{(21)}$ il a aussi une fonction plus individualisante où "l'aspect relationnel est privilégié dans l'acte d'enseigner ». ${ }^{(22)}$

Pour René Courtois, pour ses collaborateurs, « l'enseignement du métier, c'est une pédagogie de la vie... c'est la morale simple de chaque jour qui est 
vécue. On éduque par la bande, c’est dans le milieu naturel que la formation éducative trouve le plus de chance à s'exercer en profondeur ». ${ }^{(23)}$

Dans le concret, cette formation professionnelle, c'est une dizaine d'ateliers de formation industrielle (ajustage, tournage, mécanique rurale, serrurerie, menuiserie, charronnage, maçonnerie, ciment armé, peinture, plâtrerie, dessin industriel) mais aussi plusieurs ateliers agricoles (élevage, sylviculture, viticulture, laiterie, horticulture) où l'on envoie les jeunes d'un niveau plus faible ; les plus instables étant placés à l'élevage ou à la bergerie. Pour ceux qui réussissent mal dans les ateliers, le placement artisanal dans une famille est l'amorce de la vie normale. Les élèves qui ne peuvent prétendre au certificat d'aptitude professionnelle (CAP) ont la possibilité de passer un certificat d'aptitude aux métiers décernés par l'institution.

En 1945, 50 élèves sont présentés au CAP, 46 sont reçus ; en 1955, dix ans plus tard, la proportion de réussite est encore haute, sur 80 présentés, 52 obtiennent le CAP.

\section{Le séjour à Saint-Maurice}

Les jeunes qui relèvent de l'ordonnance du 2 février 1945 sur l'enfance délinquante y sont envoyés par les juges des enfants. Ils y passent deux à trois ans, le temps d'une formation professionnelle. Il est à remarquer que le contact avec les familles et le milieu d'origine est très limité. Les élèves écrivent régulièrement à leurs parents mais ils les voient rarement hors des grandes vacances.

René Courtois dit à ce sujet :

«Les familles sont insouciantes, idéalisées par les jeunes, ce n'est pas bon de faire croire à un jeune qu'il peut compter sur sa famille, si ce n'est pas vrai. "Les familles sont en général contentes que leurs garçons soient ici et s’ils ne les prennent pas en vacances, ils participent à des camps ou peuvent aller dans des familles d'accueil... " (24)

L'idée très forte de l'époque est que la famille est souvent responsable de la délinquance, de l'inadaptation du jeune et qu'il faut distendre les liens au maximum. C'est l'institution qui devient substitut parental.

$\mathrm{Au}$ lendemain de la guerre, le régime de Saint-Maurice reste encore dur. Survivance de l'Administration pénitentiaire, l'institution est partagée en trois

(24) Entretien de Jacques Bouquin avec René Courtois, op. cit. 
grandes sections de vie, c'est ce qu'on appelle le système progressif. On distingue la section " épreuve ", la section " normale ", la section " mérite ". En fonction de l'amélioration de son comportement, le jeune peut passer progressivement de la section la plus dure (section «épreuve ») à la section «normale " puis " mérite ». Il n'est pas exclu qu'il fasse le voyage inverse si son comportement régresse.

- La section « épreuve " (120 places)

Vers 1945-1950, tout arrivant y est envoyé. Cette section est composée de 5 groupes de 24 élèves, chaque groupe se trouvant sous la responsabilité d'un ou deux éducateurs. On est en Sologne, à la lisière d'une forêt, chaque groupe porte un nom d'arbre (les bouleaux, les palmiers, les acacias, les sapins, les peupliers). Ce dernier groupe quasi disciplinaire reçoit les jeunes les plus difficiles, les fugueurs... Pour la grande majorité des jeunes, ce temps d'épreuve, c'est le temps de la première année. Les jeunes couchent dans des cages à poules qui ne disparaîtront que vers 1960, on les appelle aussi " cabines ».

Tout un système de bons points que l'on donne et que l'on retire permet de mesurer les progrès d'un jeune dans les divers domaines (attitude morale, travaux manuels, travaux scolaires, performances sportives). Les notes obtenues sont affichées dans le groupe, ceci dans un but d'émulation.

- La section "normale" (60 places)

Il y a deux groupes, le groupe « excellence » (32 places) et le groupe " honneur" (28 places). On y accède en deuxième année "si on a fait preuve de gages d'amendement ». La tenue vestimentaire change, le jeune porte un veston droit avec revers, chemise et cravate. Il a un pouvoir d'achat plus grand. Il vit en dortoir et a la possibilité d'obtenir des permissions si ses parents présentent les garanties suffisantes.

- La section "mérite " (40 places)

On distingue la " mérite " (28 places) et la "super mérite" (12 places). Cette section fondée sur la confiance est réservée à ceux dont « la réadaptation sociale est en bonne voie ». Ils bénéficient de chambrettes individuelles avec lavabo. La "super mérite " bénéficie en plus d'un pavillon autonome à 500 mètres des autres groupes. Il y a un seul éducateur pour les deux sections. Les jeunes ont la clé de leur chambre, ils peuvent sortir librement le dimanche et disposent d'un costume de ville. 
Il s'agit dans cette troisième étape du système progressif d'une sorte de reconditionnement à la liberté.

Malgré son aspect novateur, le système progressif reste encore imprégné de l'esprit pénitentiaire, surtout pour la moitié des jeunes de l'institution qui sont dans des groupes épreuves - la notion d'amendement est vague. Dans les années 1930, le système progressif favorisait plus à l'intérieur des maisons d'éducation surveillée la promotion des petits caïds que celle des mineurs méritants.

Vincent Hourcq et René Courtois dès 1938 auront à affronter ce problème, le pavillon "super mérite " de 1939 sera un premier pas vers d'autres objectifs, en particulier celui de la responsabilisation du jeune.

\section{« La république " de Saint-Maurice}

L'idée de self government avait été introduite dans la formation des moniteurs-éducateurs à Fresnes en 1936 par le directeur de l'école des Roches, une école pour jeunes fils de familles qui fonctionnait sur un modèle très anglais. Ce système fondé sur la confiance, la responsabilisation, c'est aussi pour René Courtois un moyen d'associer les élèves à la gestion de l'ordre et de la discipline dans l'établissement, c'est créer un esprit nouveau où l'idée de citoyenneté du jeune n'est pas absente.

L'idée lui était venue dès 1944 en essayant d'associer les élèves de SaintMaurice à la répartition des tickets de ravitaillement.

«La république » de Saint-Maurice est instituée le 5 décembre 1945. Le document original à la belle calligraphie mentionne les buts : "Améliorer la vie à l'intérieur de l'école, maintenir et améliorer l'esprit de l'école. " (25)

Le document est signé par le directeur et les neuf membres du gouvernement (un président, un vice-président, un trésorier, des délégués aux loisirs, aux sports) sont élus par les élèves des sections « normale " et " mérite " après une campagne électorale.

Les élèves de la section "épreuve " (120 élèves) font partie de « la république ", mais ils n'ont pas le droit de vote. Il leur faudra le conquérir par leur conduite et leur passage en section " normale».

«La république » fonctionnera jusqu’en 1957, date du départ de René Courtois ; elle tiendra 450 sessions (réunions) pendant cette période et rencontrera le directeur chaque mois pour lui soumettre les points à aborder.

\section{(25) Fonds René}

Courtois, Conservatoire des archives de la protection de l'enfance et de l'adolescence, Archives du monde du travail, Roubaix. 
(26) Livre d'or de

Saint-Maurice.

(27) La collection complète du journal Espère a été déposée au Centre des archives contemporaines, Fontainebleau (fonds Brisset, versement $\left.\mathrm{n}^{\circ} 20000111\right)$
La première rencontre le 9 décembre 1945 aboutit à l'achat d'un poste de radio pour l'école. La seconde se termine par l'achat d'un cinéma $16 \mathrm{~mm}$. La troisième débouchera sur l'organisation d'une fête à Lamotte-Beuvron pour les femmes de prisonniers (février 1946).

En février 1948, le gouvernement interviendra avec efficacité pour la diminution des sanctions de cellule.

En avril 1948, "la république " désigne deux élèves, délégués de SaintMaurice, pour participer au congrès départemental à Blois du GFEN (Groupe français d'éducation nouvelle).

Progressivement " la république " interviendra dans le choix des activités et pourra donner son avis sur les méthodes.

On parle de "système socio-pédagogique ", tout cela n'est pas sans lien avec le mouvement des républiques d'enfants très développé au lendemain de la guerre, jusque dans les milieux de la rééducation.

Comme le disait, non sans humour, un inspecteur de l'Éducation surveillée, Paul Lutz : "La république” de Saint-Maurice est belle sous l'empire invisible de son directeur. " ${ }^{(26)}$

Le journal Espère, ${ }^{(27)}$ dont le premier numéro paraît en janvier 1945 et qui se poursuivra jusqu'au début des années 1970, sera très vite l'organe officiel de " la république ".

Largement diffusé à l'extérieur, il permet de montrer à l'environnement une autre image que celle de l'ancienne colonie pénitentiaire encore si proche et diffuse largement tous les résultats sportifs où Saint-Maurice brille souvent dans les championnats départementaux.

Le système progressif va disparaître au début des années 1950. L’idée se fait de plus en plus forte qu'un adolescent ne peut avoir une progression linéaire et que la notion d'amendabilité trop vague peut amener à une confusion entre moralité et conformisme social. Si l'isolement est maintenu (4 cellules), c'est la sanction la plus grave. Le groupe disciplinaire " les Peupliers » disparaît en 1952. On ne parle plus de section d'épreuve, elle est remplacée par une progressivité horizontale à l'intérieur du groupe.

Chaque jeune est affecté à un groupe éducatif suivant sa personnalité ou son degré de maturité. Subsistent quelques groupes sans éducateurs auxquels on a conservé les dénominations : " honneur ", " mérite ", "super mérite ». 
C'est un nouveau stade de la rééducation qui s'élabore au début des années 1950 avec une priorité plus grande apportée à chaque jeune. On ne peut comprendre le Saint-Maurice de cette époque sans faire référence au charisme, à la forte personnalité non sans contrastes de René Courtois.

Il est le père fondateur, le chef à la fois redouté et incontesté, il sait s'entourer mais tout passe par lui. "La république », le "super mérite " ne sont possibles que parce qu'il est omniprésent, reconnu.

Il a la trempe des novateurs. Sa force de caractère et sûrement son amour des jeunes ont été les éléments majeurs de sa réussite dans la réforme de Saint-Maurice. 